\title{
Calculated Optimized Structure and Geometric Analysis of Oxygenated Fuel Additives: Alcohols and Ethers
}

\author{
Sinem Gurkan Aydin \\ Opticianry Department \\ Vocational School of Health Services \\ Istanbul Gelisim University \\ Istanbul, Turkey \\ sgurkan@gelisim.edu.tr
}

Arzu Ozgen

Medical Laboratory Techniques Department

Vocational School of Health Services

Istanbul Gelisim University

Istanbul,Turkey

aozgen@gelisim.edu.tr

\author{
Oktay Polat \\ Aircraft Mechanic-Engine Department \\ School of Applied Sciences \\ Istanbul Gelisim University \\ Istanbul,Turkey \\ 190809911@ogr.gelisim.edu.tr \\ Emre Turali \\ Aircraft Mechanic-Engine Department \\ School of Applied Sciences \\ Istanbul Gelisim University \\ Istanbul,Turkey \\ 190809907@ogr.gelisim.edu.tr
}

\begin{abstract}
Oxygenated fuel additives are added to gasoline in order to reduce the gases released from vehicle engines, to increase the octane number, and to expand the use of renewable resources. In this study, molecular and geometric analysis of oxygenated fuel additives was conducted theoretically and the energy values of optimized structures were calculated. The effect of molecular energy and the bond structure between $\mathrm{C}, \mathrm{H}$ and $\mathrm{O}$ on the chemical and physical properties of some oxygen fuel additives were investigated. The obtained results will form the basis for future studies in obtaining more environmentally friendly fuels.
\end{abstract}

Keywords-oxygenated fuel additives; gasoline; octane booster; energy; molecular analysis; geometric analysis

\section{INTRODUCTION}

Energy plays an important role in our daily life and is important for the socio-economic development [1]. Today, the dominant share of fossil fuels in energy consumption continues $[2,3]$. In $2011,33 \%$ of the energy consumed was supplied from oil, $28 \%$ from coal, $22 \%$ from natural gas, and $6 \%$ from nuclear energy while the remaining $11 \%$ was covered by other energy sources [4-7]. Petroleum fuels are widely used in the transportation sector. In $2011,54 \%$ of world oil consumption was in the transportation sector, $18 \%$ in the industrial sector, $11 \%$ in the domestic, commercial and agricultural areas, $10 \%$ in the petrochemical industry, and $7 \%$ was consumed in electricity production [8-10].

It is inevitable to start producing more environmentally friendly fuels by changing the properties of the existing additives. A detailed examination of the molecular structure of the substances added to fuels will shed light on further studies.
Oxygen fuel additives are organic substances that contain one or more oxygen atoms in their molecular structures. Oxygenates consist of different alcohols, ethers, esters and carbonates, but primary alcohols (methanol, ethanol) and ethers are commercially used oxygenates [11]. Alcohols and ethers have been used for a long time to increase the octane number of gasoline, reduce the vapor pressure and add oxygen content to the mix. Alcohols, heavy molecular alcohols, and ethers like methanol, ethanol, isopropyl alcohol, tert-butyl alcohol (TBA), tert-amyl alcohol (TAA), propanol, butanol, pentanol, methyl tertiary butyl ether (MTBE), 2-methoxy-2-methylpropane (ETBE), tert-butyl ethylether (TAME), 2-ethoxy-2methylbutane (TAEE), 2-ethoxy-2-methybutane (DIPE), and di-tertamyl ether (di-TAE) can be used as fuel additives. In practice, alcohols such as methanol, ethanol, butanol, MTBE, and ETBE are the most preferred oxygenated fuel additives. Since the physical and chemical properties of each alcohol and ether are different, the type and amount of the additives used directly affects the physical and chemical properties of the gasoline. Some alcohols such as ethanol and methanol can be used as both pure fuel and fuel additive [12]. There are advantages and disadvantages of using alcohol and ethers as a fuel additive [13]. The biggest advantage of alcohols is that their octane number is high. Therefore, the efficiency of the engine can be increased without knocking by selecting high compression ratios [14] while reducing $\mathrm{CO}, \mathrm{HC}$ and $\mathrm{PM}$ emissions. Due to the high hidden evaporation temperatures, they increase the density of the mixture or air taken into the cylinder, thus the volumetric efficiency of the engine [15].

Alcohols are safer in terms of transportation and storage due to their auto-ignition and flash point compared to gasoline 
[16]. Different results are available from the literature regading the effect of alcohols on $\mathrm{NO}_{\mathrm{x}}$ and $\mathrm{CO}_{2}$ emissions [17]. However, if alcohols are produced from biomass, they do not increase the total carbon dioxide emission since the carbon dioxide $\left(\mathrm{CO}_{2}\right)$ formed as a result of combustion of alcohols will be used by plants again in photosynthesis. However, the production cost of alcohols is higher than gasoline. Low heat values compared to gasoline cause lower engine power and increased fuel consumption. They have high water-solubility rates and are highly prone to absorbing moisture from the air [18]. Therefore, phase separation occurs in the gasoline-alcohol mixture and the corrosion effect of the fuel increases [19].

The thermal value of the ethers is lower than the thermal value of gasoline, but higher than the thermal value of alcohols. The octane numbers are higher than the octane number of gasoline but lower than the octane number of alcohols. The ethers' oxygen content is lower than alcohols'. However, ethers have some important advantages over alcohols. Ethers have low Reid vapor pressure and therefore do not increase volatile hydrocarbon emissions [20]. They mix with gasoline at any rate and phase separation does not occur. Thanks to their oxygen content, they reduce $\mathrm{CO}, \mathrm{HC}$ and $\mathrm{PM}$ emissions. However, their production cost is high and they can contaminate underground drinking water by seeping through storage tanks [21]. Ethers are produced by the reaction of alcohols and petroleum-based hydrocarbons accompanied by an acidic catalyst. MTBE is obtained by reacting methanol and isobuten, an oil-based hydrocarbon, with an acidic catalyst at high pressure and temperature [22]. When using ethanol instead of methanol with the same method, ETBE is obtained, which is a partially renewable fuel additive since ethanol from biomass is used. At the end of the reaction, a mixture of ETBE and ethanol azeotropic is formed. It is not possible to separate ETBE from this mixture by simple distillation. For separation, first of all, the azeotropic molecules formed by adding water to the reaction medium must be broken down and then the isobutene, ethanol and water must be removed and the ETBE must be purified [23], thus its production cost increases [20, 23]. It is obtained by reacting TAME with isoamylene (C5 olefins), a hydrocarbon, in the presence of an acidic catalyst. TAEE is obtained if ethanol is used in this reaction instead of methanol. It is a partially renewable oxygen fuel additive since ethanol obtained by fermentation of biomass is used in TAEE production [24]. DIPE production is different from the production of other ethers. Alcohol is not used in DIPE production. Isopropyl alcohol is obtained by propylene. Then, isopropyl alcohol reacts with propylene to obtain diisopropyl alcohol. The biggest disadvantage of DIPE is that when it is mixed with gasoline, peroxides that can explode easily occur and therefore pose more risks than other ethers during transportation, storage and distribution [22]. Di-TAE is obtained by reacting isoamylene with isoamyl alcohol [24]. Isoamyl alcohol is approximately the $60 \%$ of the fusel oil and appears as a by-product in ethanol production [25]. Di-TAE has the potential to be used as an oxygenated fuel due to its cheap raw material production and being partially renewable [26].

The basic state optimized structures of some oxygenic fuel additives were calculated at the level of $6-311++\mathrm{G}(\mathrm{d}, \mathrm{p})$ with
DFT (Density Functional Theory) (B3LYP) method in this study. Methanol, ethanol, MTBE, ETBE, TAEE, TAME and DIPE were used as fuel additives. In addition, the geometrical parameters of the fuel additives were determined and detailed and a comparison of their physical and chemical properties was made. The effects of molecular energy and bond structure between $\mathrm{C}, \mathrm{H}$ and $\mathrm{O}$ on the chemical and physical properties of some oxygen fuel additives were investigated.

\section{MATERIALS AND METHODS}

Optimized basic state conformations of all oxygenic fuel additives were calculated by using spin-unrestricted DFT (B3LYP) method with 6-311++G (d,p) basis sets implemented in the polarizable continuum model (PCM). The reason for choosing this method is that it gives better results in molecular calculations of a similar structure [27, 28]. Gaussian 03 [29] and Gauss-View molecular visualization [30] were used in all calculations on a personal computer. Potential Energy Surface (PES) scans of oxygen fuel additives showed one or more minimum-energy structures. These minimum structures were chosen to achieve even more stable ones and more accurate results.

\section{RESULTS AND DISCUSSION}

The structures of molecules can be determined in detail using molecular modeling methods. This will be a guide for new materials to be developed in the future or for new materials to be added. The calculated ground state optimized structures and energies of the all oxygenic fuel additives are shown in Figure 1. At the same time, molecular energy calculations for all fuel additives were made in the basic set of 6-311 + G (d, p) with the DFT (B3LYP) method. The atomic bonds and energy values of the fuel additives in the molecular structure vary as shown in Figure 1. This difference reflects on many physical and chemical properties (fuel performance, engine octane number, flash point, boiling point, freezing point, viscosity, etc.).

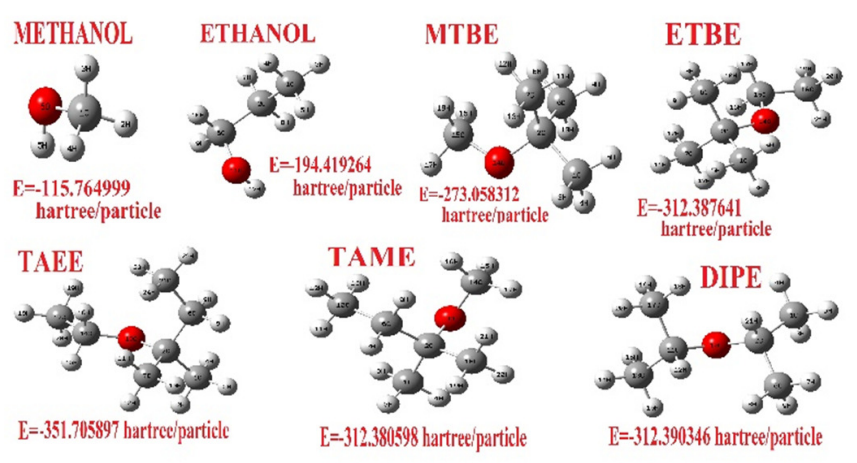

Fig. 1. The calculated ground state optimized structures and energies of the all oxygenic fuel additives

\section{A. Methanol}

Methanol is a high extract fuel additive. The octane value of methanol is ranges from 105 to 110 . Evaporation heat and extract value play important roles in the characteristics of the internal combustion engine using this fuel. As seen in the 
molecular structure of methanol, it is a fuel additive that contains oxygen. Therefore, it is important to know the binding properties of oxygen in its molecular structure with other atoms. Some geometric parameters and energy of methanol were calculated in this study. Geometrical parameters such as bond length, bond angle, and torsion angle calculated at the B3LYP/6311++G(d, p) level of theory are given in Table I.

TABLE I. SELECTED GEOMETRICAL PARAMETERS OF METHANOL*

\section{\begin{tabular}{|c|c|c|}
\hline Dihedral angle $\left({ }^{\circ}\right)$ & Angle $\left(^{\circ}\right)$ & Bond $(\lambda)$ \\
\hline
\end{tabular}} \begin{tabular}{|l|l|l|l|l|l|}
\hline $5(\mathrm{H})-6(\mathrm{O})-1(\mathrm{C})-3(\mathrm{H})$ & 179.999 & $5(\mathrm{H})-6(\mathrm{O})-1(\mathrm{C})$ & 108.833 & $6(\mathrm{O})-5(\mathrm{H})$ & 0.961 \\
\hline
\end{tabular} \begin{tabular}{|l|l|l|l|l|l|l|}
\hline $5(\mathrm{H})-6(\mathrm{O})-1(\mathrm{C})-4(\mathrm{H})$ & -61.475 & $6(\mathrm{O})-1(\mathrm{C})-3(\mathrm{H})$ & 106.661 & $6(\mathrm{O})-1(\mathrm{C})$ & 1.424 \\
\hline
\end{tabular} \begin{tabular}{|l|l|l|l|l|l|}
\hline $3(\mathrm{H})-1(\mathrm{C})-6(\mathrm{O})-5(\mathrm{H})$ & 179.999 & $4(\mathrm{H})-1(\mathrm{C})-3(\mathrm{H})$ & 108.431 & $4(\mathrm{H})-1(\mathrm{C})$ & 1.096
\end{tabular} \begin{tabular}{|l|l|l|l|l|l|}
\hline $4(\mathrm{H})-1(\mathrm{C})-6(\mathrm{O})-5(\mathrm{H})$ & -61.475 & $3(\mathrm{H})-1(\mathrm{C})-4(\mathrm{H})$ & 108.431 & $4(\mathrm{H})-1(\mathrm{C})$ & 1.096 \\
\hline
\end{tabular} ${ }^{*}$ Computed at DFT(B3LYP)/6311++G(d,p) level

\section{B. Ethanol}

The octane value of ethanol was determined between about 92-108. Ethanol is a non-toxic substance that dissolves in water and can quickly biodegrade and disappear. It also significantly reduces the carbon monoxide rate. Ethanol is a two-carbon monoalcohol. Geometric analysis of carbon atoms in the molecular structure was conducted, and the same geometrical parameters were calculated and are given in Table II.

TABLE II. SELECTED GEOMETRICAL PARAMETERS OF ETHANOL

\begin{tabular}{|c|c|c|c|c|c|}
\hline \multicolumn{2}{|c|}{ Dihedral angle $\left(^{\circ}\right)$} & \multicolumn{2}{c|}{ Angle } & \multicolumn{2}{c|}{ Bond } \\
\hline $\begin{array}{c}12(\mathrm{H})-11(\mathrm{O})- \\
6(\mathrm{C})-9(\mathrm{H})\end{array}$ & 59.433 & $12(\mathrm{H})-11(\mathrm{O})-6(\mathrm{C})$ & 108.481 & $12(\mathrm{H})-11(\mathrm{O})$ & 0.962 \\
\hline $\begin{array}{c}12(\mathrm{H})-11(\mathrm{O})- \\
6(\mathrm{C})-10(\mathrm{H})\end{array}$ & 175.032 & $10(\mathrm{H})-6(\mathrm{C})-11(\mathrm{O})$ & 105.109 & $11(\mathrm{O})-6(\mathrm{C})$ & 1.429 \\
\hline $\begin{array}{c}11(\mathrm{O})-6(\mathrm{C})-2(\mathrm{C})- \\
8(\mathrm{H})\end{array}$ & 61.470 & $11(\mathrm{O})-6(\mathrm{C})-10(\mathrm{H})$ & 105.109 & $9(\mathrm{H})-6(\mathrm{C})$ & 1.098 \\
\hline $\begin{array}{c}11(\mathrm{O})-6(\mathrm{C})-2(\mathrm{C})- \\
7(\mathrm{H})\end{array}$ & 176.102 & $6(\mathrm{C})-2(\mathrm{C})-8(\mathrm{H})$ & 108.912 & $10) \mathrm{H})-6(\mathrm{C})$ & 1.093 \\
\hline $\begin{array}{c}6(\mathrm{C})-2(\mathrm{C})-1(\mathrm{C})- \\
3(\mathrm{H})\end{array}$ & 178.227 & $8(\mathrm{H})-2(\mathrm{C})-7(\mathrm{H})$ & 105.967 & $6(\mathrm{C})-2(\mathrm{C})$ & 1.528 \\
\hline $\begin{array}{c}6(\mathrm{C})-2(\mathrm{C})-1(\mathrm{C})- \\
4(\mathrm{H})\end{array}$ & -61.869 & $2(\mathrm{C})-1(\mathrm{C})-3(\mathrm{H})$ & 111.221 & $2(\mathrm{C})-1(\mathrm{C})$ & 1.530 \\
\hline $\begin{array}{c}6(\mathrm{C})-2(\mathrm{C})-1(\mathrm{C})- \\
5(\mathrm{H})\end{array}$ & 57.727 & $6(\mathrm{C})-2(\mathrm{C})-1(\mathrm{C})$ & 113.666 & $2(\mathrm{C})-8(\mathrm{H})$ & 1.098 \\
\hline $\begin{array}{c}11(\mathrm{O})-6(\mathrm{C})-2(\mathrm{C})- \\
1(\mathrm{C})\end{array}$ & -61.469 & $3(\mathrm{H})-1(\mathrm{C})-4(\mathrm{H})$ & 107.759 & $2 \mathrm{C}-7 \mathrm{H}$ & 1.096 \\
\hline
\end{tabular}

\section{C. $M T B E$}

MTBE is a water-soluble, volatile, and colorless fuel additive, commonly used to increase octane number. It is also known to dilute fuel better by gasoline components. When mixed with soil, it can biodegrade under the influence of bacteria. MTBE has an octane number between 101-116. The oxygen content in its molecular structure is $16 \%$ by mass and its geometrical analysis can be seen in Table III.

\section{ETBE}

ETBE is produced from ethanol and isobutylene. Although it improves the combustion properties of the fuel, it is more compatible than ethanol. It provides high air quality when added to fuel. Although ETBE has the highest octane number among the oxygenated fuel additives mentioned in this study, it was found to have the highest molecular energy in our calculations. ETBE is an important fuel additive with superior properties and its geometric analysis is given in Table IV.

TABLE III. SELECTED GEOMETRICAL PARAMETERS OF MTBE*

\begin{tabular}{|cc|c|c|c|c|}
\hline \multicolumn{2}{|c|}{ Dihedral angle $\left.{ }^{\circ}\right)$} & \multicolumn{2}{c|}{ Angle } & \multicolumn{2}{c|}{ Bond } \\
\hline $\begin{array}{c}18(\mathrm{H})-15(\mathrm{C})-14(\mathrm{O})- \\
2(\mathrm{C})\end{array}$ & -61.683 & $\begin{array}{c}17(\mathrm{H})-15(\mathrm{C})- \\
14(\mathrm{O})\end{array}$ & 106.071 & $18(\mathrm{H})-15(\mathrm{C})$ & 1.097 \\
\hline $\begin{array}{c}17(\mathrm{H})-15(\mathrm{C})-14(\mathrm{O})- \\
2(\mathrm{C})\end{array}$ & -179.985 & $\begin{array}{c}15(\mathrm{C})-14(\mathrm{O})- \\
2(\mathrm{C})\end{array}$ & 118.169 & $15(\mathrm{C})-14(\mathrm{O})$ & 1.415 \\
\hline $\begin{array}{c}15(\mathrm{C})-14(\mathrm{O})-2(\mathrm{C})- \\
7(\mathrm{C})\end{array}$ & 61.905 & $\begin{array}{c}14(\mathrm{O})-2(\mathrm{C})- \\
7(\mathrm{C})\end{array}$ & 110.897 & $14(\mathrm{O})-2(\mathrm{C})$ & 1.446 \\
\hline $\begin{array}{c}15(\mathrm{C})-14(\mathrm{O})-2(\mathrm{C})- \\
6(\mathrm{C})\end{array}$ & -61.821 & $\begin{array}{c}14(\mathrm{O})-2(\mathrm{C})- \\
6(\mathrm{C})\end{array}$ & 110.903 & $2(\mathrm{C})-7(\mathrm{C})$ & 1.536 \\
\hline $\begin{array}{c}15(\mathrm{C})-14(\mathrm{O})-2(\mathrm{C})- \\
1(\mathrm{C})\end{array}$ & -179.959 & $\begin{array}{c}14(\mathrm{O})-2(\mathrm{C})- \\
1(\mathrm{C})\end{array}$ & 103.402 & $2(\mathrm{C})-6(\mathrm{C})$ & 1.536 \\
\hline $\begin{array}{c}14(\mathrm{O})-2(\mathrm{C})-7(\mathrm{C})- \\
12(\mathrm{H})\end{array}$ & -67.712 & $\begin{array}{c}12(\mathrm{H})-7(\mathrm{C})- \\
2(\mathrm{C})\end{array}$ & 112.021 & $2(\mathrm{C})-1(\mathrm{C})$ & 1.530 \\
\hline $\begin{array}{c}14(\mathrm{O})-2(\mathrm{C})-6(\mathrm{C})- \\
9(\mathrm{H})\end{array}$ & -172.738 & $\begin{array}{c}4(\mathrm{H})-1(\mathrm{C})- \\
2(\mathrm{C})\end{array}$ & 110.196 & $12(\mathrm{H})-7(\mathrm{C})$ & 1.092 \\
\hline $\begin{array}{c}14(\mathrm{O})-2(\mathrm{C})-1(\mathrm{C})- \\
4(\mathrm{H})\end{array}$ & 59.671 & $\begin{array}{c}9(\mathrm{H})-6(\mathrm{C})- \\
2(\mathrm{C})\end{array}$ & 110.184 & $9(\mathrm{H})-6(\mathrm{C})$ & 1.093 \\
\hline $\begin{array}{c}12(\mathrm{H})-7(\mathrm{C})-2(\mathrm{C})- \\
1(\mathrm{C})\end{array}$ & 178.385 & $\begin{array}{c}6(\mathrm{C})-2(\mathrm{H})- \\
1(\mathrm{C})\end{array}$ & 110.234 & $4(\mathrm{H})-1(\mathrm{C})$ & 1.092 \\
\hline $\begin{array}{c}12(\mathrm{H})-7(\mathrm{C})-2(\mathrm{C})- \\
6(\mathrm{C})\end{array}$ & 55.996 & $\begin{array}{c}2(\mathrm{C})-6(\mathrm{C})- \\
8(\mathrm{H})\end{array}$ & 112.024 & & \\
\hline $\begin{array}{r}7(\mathrm{C})-2(\mathrm{C})-1(\mathrm{C})- \\
4(\mathrm{H})\end{array}$ & 178.266 & $\begin{array}{c}2(\mathrm{C})-1(\mathrm{C})- \\
3(\mathrm{H})\end{array}$ & 110.196 & & \\
\hline $\begin{array}{r}7(\mathrm{C})-2(\mathrm{C})-6(\mathrm{C})- \\
9(\mathrm{H})\end{array}$ & 63.555 & & & \\
\hline
\end{tabular}

TABLE IV. SELECTED GEOMETRICAL PARAMETERS OF ETBE*

\begin{tabular}{|c|c|c|c|c|c|}
\hline \multicolumn{2}{|c|}{ Dihedral angle $\left.{ }^{\circ}\right)$} & \multicolumn{2}{c|}{ Angle } & \multicolumn{2}{c|}{ Bond } \\
\hline $\begin{array}{c}21(\mathrm{H})-18(\mathrm{C})-15(\mathrm{C})- \\
14(\mathrm{O})\end{array}$ & 59.955 & $\begin{array}{c}20(\mathrm{H})-18(\mathrm{C})- \\
15(\mathrm{C})\end{array}$ & 110.601 & $21(\mathrm{H})-18(\mathrm{C})$ & 1.092 \\
\hline $\begin{array}{c}20(\mathrm{H})-18(\mathrm{C})-15(\mathrm{C})- \\
14(\mathrm{O})\end{array}$ & -59.959 & $\begin{array}{c}18(\mathrm{C})-15(\mathrm{C})- \\
16(\mathrm{H})\end{array}$ & 109.928 & $18(\mathrm{C})-15(\mathrm{C})$ & 1.520 \\
\hline $\begin{array}{c}19(\mathrm{H})-18(\mathrm{C})-15(\mathrm{C})- \\
14(\mathrm{O})\end{array}$ & 179.998 & $\begin{array}{c}18(\mathrm{C})-15(\mathrm{C})- \\
17(\mathrm{H})\end{array}$ & 109.928 & $15(\mathrm{C})-16(\mathrm{H})$ & 1.098 \\
\hline $\begin{array}{c}18(\mathrm{C})-15(\mathrm{C})-14(\mathrm{O})- \\
2(\mathrm{C})\end{array}$ & 179.980 & $\begin{array}{c}18(\mathrm{C})-15(\mathrm{C})- \\
14(\mathrm{O})\end{array}$ & 107.687 & $15(\mathrm{C})-14(\mathrm{O})$ & 1.421 \\
\hline $\begin{array}{c}16(\mathrm{H})-15(\mathrm{C})-14(\mathrm{O})- \\
2(\mathrm{C})\end{array}$ & -59.736 & $\begin{array}{c}15(\mathrm{C})-14(\mathrm{O})- \\
2(\mathrm{C})\end{array}$ & 118.745 & $14(\mathrm{O})-2(\mathrm{C})$ & 1.445 \\
\hline $\begin{array}{c}17(\mathrm{H})-15(\mathrm{C})-14(\mathrm{O})- \\
2(\mathrm{C})\end{array}$ & 59.696 & $14(\mathrm{O})-2(\mathrm{C})-1(\mathrm{C})$ & 103.445 & $2(\mathrm{C})-1(\mathrm{C})$ & 1.530 \\
\hline $\begin{array}{c}7(\mathrm{C})-2(\mathrm{C})-14(\mathrm{O})- \\
15(\mathrm{C})\end{array}$ & 61.865 & $14(\mathrm{O})-2(\mathrm{C})-7(\mathrm{C})$ & 110.964 & $2(\mathrm{C})-7(\mathrm{C})$ & 1.536 \\
\hline $7(\mathrm{C})-2(\mathrm{C})-6(\mathrm{C})-9(\mathrm{H})$ & 63.806 & $2(\mathrm{C})-7(\mathrm{C})-12(\mathrm{H})$ & 112.046 & $7(\mathrm{C})-13(\mathrm{H})$ & 1.093 \\
\hline $7(\mathrm{C})-2(\mathrm{C})-1(\mathrm{C})-4(\mathrm{H})$ & 178.315 & $2(\mathrm{C})-1(\mathrm{C})-4(\mathrm{H})$ & 110.182 & $1(\mathrm{C})-3(\mathrm{H})$ & 1.092 \\
\hline $9(\mathrm{H})-6(\mathrm{C})-2(\mathrm{C})-14(\mathrm{O})$ & -172.388 & $14(\mathrm{O})-2(\mathrm{C})-6(\mathrm{C})$ & 110.962 & $6(\mathrm{C})-10(\mathrm{H})$ & 1.093 \\
\hline $\begin{array}{c}6(\mathrm{C})-2(\mathrm{C})-14(\mathrm{O})- \\
15(\mathrm{C})\end{array}$ & -61.899 & $2(\mathrm{C})-6(\mathrm{C})-9(\mathrm{H})$ & 110.179 & $2(\mathrm{C})-6(\mathrm{C})$ & 1.536 \\
\hline & $2(\mathrm{C})-6(\mathrm{H})-10(\mathrm{H})$ & 110.298 & $7(\mathrm{C})-13(\mathrm{H})$ & 1.093 \\
\hline
\end{tabular}

\section{E. TAEE}

TAEE is a fuel additive belonging to the ether class and its molecular formula is $\mathrm{C}_{7} \mathrm{H}_{16} \mathrm{O}$. The engine octane number of TAEE has been determined to be above 105. The calculated geometrical analysis results of TAEE are given in Table $\mathrm{V}$.

\section{F. TAME}

It is a fuel additive produced from naphtha $\mathrm{C} 4$ distillation fractions. It is added to the fuels in order to increase their 
octane level, change the tetraethyl lead and increase oxygen content. It has a high boiling point and a low freezing point. The geometrical analysis of TAME is given in Table VI.

TABLE V. SELECTED GEOMETRICAL PARAMETERS OF TAEE*

\begin{tabular}{|c|c|c|c|c|c|}
\hline \multicolumn{2}{|c|}{ Dihedral angle $\left({ }^{\circ}\right)$} & \multicolumn{2}{|l|}{ Angle } & \multicolumn{2}{|l|}{ Bond } \\
\hline $\begin{array}{l}18(\mathrm{H})-17(\mathrm{C})- \\
14(\mathrm{O})-13(\mathrm{O})\end{array}$ & 178.613 & $18(\mathrm{H})-17(\mathrm{C})-14(\mathrm{C})$ & 110.285 & $18(\mathrm{H})-17(\mathrm{C})$ & 1.093 \\
\hline $\begin{array}{l}20(\mathrm{H})-17(\mathrm{C})- \\
14(\mathrm{C})-13(\mathrm{O})\end{array}$ & 58.628 & $17(\mathrm{C})-14(\mathrm{C})-13(\mathrm{O})$ & 107.394 & $17(\mathrm{C})-14(\mathrm{C})$ & 1.519 \\
\hline $\begin{array}{l}19(\mathrm{H})-17(\mathrm{C})- \\
14(\mathrm{C})-13(\mathrm{O})\end{array}$ & -61.228 & $16(\mathrm{H})-14(\mathrm{C})-13(\mathrm{O})$ & 111.481 & $15(\mathrm{H})-14(\mathrm{C})$ & 1.097 \\
\hline $\begin{array}{c}17(\mathrm{C})-14(\mathrm{C})- \\
13(\mathrm{O})-2(\mathrm{C})\end{array}$ & 174.778 & $14(\mathrm{C})-13(\mathrm{O})-2(\mathrm{C})$ & 120.671 & $14(\mathrm{C})-13(\mathrm{O})$ & 1.423 \\
\hline $\begin{array}{c}16(\mathrm{H})-14(\mathrm{C})- \\
13(\mathrm{O})-2(\mathrm{C})\end{array}$ & 54.219 & $13(\mathrm{O})-2(\mathrm{C})-7(\mathrm{C})$ & 112.997 & $13(\mathrm{O})-2(\mathrm{C})$ & 1.450 \\
\hline $\begin{array}{l}15(\mathrm{H})-14(\mathrm{C})- \\
13(\mathrm{O})-2(\mathrm{C})\end{array}$ & -65.338 & $13(\mathrm{O})-2(\mathrm{C})-6(\mathrm{C})$ & 109.403 & $2(\mathrm{C})-1(\mathrm{C})$ & 1.534 \\
\hline $\begin{array}{c}\text { 14(C)-13(O)- } \\
2(\mathrm{C})-6(\mathrm{C})\end{array}$ & -98.065 & $13(\mathrm{O})-2(\mathrm{C})-1(\mathrm{C})$ & 103.982 & $2(C)-7(C)$ & 1.535 \\
\hline $\begin{array}{c}\text { 14(C)-13(O)- } \\
2(\mathrm{C})-1(\mathrm{C})\end{array}$ & 145.939 & $7(\mathrm{C})-2(\mathrm{C})-1(\mathrm{C})$ & 109.407 & $11(\mathrm{H})-7(\mathrm{C})$ & 1.091 \\
\hline $\begin{array}{c}\text { 14(C)-13(O)- } \\
2(\mathrm{C})-7(\mathrm{C})\end{array}$ & 27.405 & $7(C)-2(C)-6(C)$ & 111.964 & $4(\mathrm{H})-1(\mathrm{C})$ & 1.092 \\
\hline $\begin{array}{c}13(\mathrm{O})-2(\mathrm{C})- \\
6(\mathrm{C})-8(\mathrm{H})\end{array}$ & -174.488 & $1(C)-2(C)-6(C)$ & 108.716 & $2(C)-6(C)$ & 1.547 \\
\hline $\begin{array}{l}13(\mathrm{O})-2(\mathrm{C})- \\
6(\mathrm{C})-21(\mathrm{C})\end{array}$ & 61.924 & $2(C)-6(C)-21(C)$ & 117.267 & $6(C)-21(C)$ & 1.531 \\
\hline $\begin{array}{c}13(\mathrm{O})-2(\mathrm{C})- \\
1(\mathrm{C})-4(\mathrm{H})\end{array}$ & 54.874 & $2(\mathrm{C})-6(\mathrm{C})-9(\mathrm{H})$ & 107.026 & $6(\mathrm{C})-8(\mathrm{H})$ & 1.096 \\
\hline $\begin{array}{c}2(\mathrm{C})-6(\mathrm{C})- \\
21(\mathrm{C})-24(\mathrm{H}) \\
\end{array}$ & 58.806 & $24(\mathrm{H})-21(\mathrm{C})-6(\mathrm{C})$ & 112.448 & $21(\mathrm{C})-24(\mathrm{H})$ & 1.093 \\
\hline $\begin{array}{c}8(\mathrm{H})-6(\mathrm{C})-2(\mathrm{C})- \\
1(\mathrm{C})\end{array}$ & -61.545 & $9(\mathrm{H})-6(\mathrm{C})-21(\mathrm{C})$ & 108.701 & $1(\mathrm{C})-5(\mathrm{H})$ & 1.093 \\
\hline $\begin{array}{c}1(\mathrm{C})-2(\mathrm{C})-6(\mathrm{C})- \\
21(\mathrm{C})\end{array}$ & 174.867 & $4(\mathrm{H})-1(\mathrm{C})-2(\mathrm{C})$ & 109.955 & $6(\mathrm{C})-9(\mathrm{H})$ & 1.095 \\
\hline
\end{tabular}

TABLE VI. SELECTED GEOMETRICAL PARAMETERS OF TAME*

\begin{tabular}{|c|c|c|c|c|c|}
\hline \multicolumn{2}{|c|}{ Dihedral angle $\left.{ }^{\circ}\right)$} & \multicolumn{3}{|c|}{ Angle } & \multicolumn{2}{c|}{ Bond } \\
\hline $\begin{array}{c}12(\mathrm{H})-10(\mathrm{C})- \\
6(\mathrm{C})-2(\mathrm{C})\end{array}$ & -175.546 & $12(\mathrm{H})-10(\mathrm{C})-6(\mathrm{C})$ & 110.441 & $12(\mathrm{H})-10(\mathrm{C})$ & 1.093 \\
\hline $\begin{array}{c}10(\mathrm{C})-6(\mathrm{C})-2(\mathrm{C})- \\
18(\mathrm{C})\end{array}$ & 177.394 & $10(\mathrm{C})-6(\mathrm{C})-2(\mathrm{C})$ & 115.513 & $10(\mathrm{C})-6(\mathrm{C})$ & 1.531 \\
\hline $\begin{array}{c}10(\mathrm{C})-6(\mathrm{C})-2(\mathrm{C})- \\
9(\mathrm{O})\end{array}$ & 54.175 & $7(\mathrm{H})-6(\mathrm{C})-2(\mathrm{C})$ & 107.567 & $8(\mathrm{H})-6(\mathrm{C})$ & 1.095 \\
\hline $\begin{array}{c}10(\mathrm{C})-6(\mathrm{C})-2(\mathrm{C})- \\
1(\mathrm{C})\end{array}$ & -60.729 & $8(\mathrm{H})-6(\mathrm{C})-2(\mathrm{C})$ & 108.756 & $6(\mathrm{C})-2(\mathrm{C})$ & 1.547 \\
\hline $\begin{array}{c}8(\mathrm{H})-6(\mathrm{C})-2(\mathrm{C})- \\
9(\mathrm{O})\end{array}$ & -60.089 & $1(\mathrm{C})-2(\mathrm{C})-9(\mathrm{O})$ & 103.411 & $1(\mathrm{C})-2(\mathrm{C})$ & 1.531 \\
\hline $\begin{array}{c}7(\mathrm{H})-6(\mathrm{C})-2(\mathrm{C})- \\
1(\mathrm{C})\end{array}$ & 62.289 & $9(\mathrm{O})-14(\mathrm{C})-15(\mathrm{H})$ & 106.103 & $1(\mathrm{C})-5(\mathrm{H})$ & 1.093 \\
\hline $\begin{array}{c}1(\mathrm{C})-2(\mathrm{C})-9(\mathrm{O})- \\
14(\mathrm{C})\end{array}$ & -176.194 & $9(\mathrm{O})-2(\mathrm{C})-18(\mathrm{C})$ & 110.962 & $2(\mathrm{C})-9(\mathrm{O})$ & 1.446 \\
\hline $\begin{array}{c}6(\mathrm{C})-2(\mathrm{C})-1(\mathrm{C})- \\
5(\mathrm{H})\end{array}$ & -56.295 & $9(\mathrm{O})-2(\mathrm{C})-6(\mathrm{C})$ & 111.161 & $2(\mathrm{C})-18(\mathrm{C})$ & 1.536 \\
\hline $\begin{array}{c}6(\mathrm{C})-2(\mathrm{C})-18(\mathrm{C})- \\
20(\mathrm{H})\end{array}$ & -175.265 & $1(\mathrm{C})-2(\mathrm{C})-18(\mathrm{C})$ & 109.660 & $18(\mathrm{C})-21(\mathrm{H})$ & 1.092 \\
\hline $\begin{array}{c}15(\mathrm{H})-14(\mathrm{C})- \\
9(\mathrm{O})-2(\mathrm{C})\end{array}$ & -178.039 & $1(\mathrm{C})-2(\mathrm{C})-6(\mathrm{C})$ & 111.621 & $9(\mathrm{O})-14(\mathrm{C})$ & 1.415 \\
\hline $\begin{array}{c}6(\mathrm{C})-2(\mathrm{C})-18(\mathrm{C})- \\
20(\mathrm{H})\end{array}$ & -175.265 & & $14(\mathrm{C})-16(\mathrm{H})$ & 1.097 \\
\hline
\end{tabular}

\section{G. $D I P E$}

It is a highly flammable fuel additive, with a serious risk of fire and explosion when kept in the air for a long time. The geometrical analysis of oxygen and carbon atoms is given in Table VII.

TABLE VII. SELECTED GEOMETRICAL PARAMETERS OF DIPE*

\begin{tabular}{|c|c|c|c|c|c|}
\hline \multicolumn{2}{|c|}{ Dihedral angle $\left(^{\circ}\right)$} & \multicolumn{3}{|c|}{ Angles } & \multicolumn{2}{c|}{ Bonds } \\
\hline $\begin{array}{c}15(\mathrm{H})-13(\mathrm{C})- \\
11(\mathrm{C})-17(\mathrm{C})\end{array}$ & 62.076 & $20(\mathrm{H})-17(\mathrm{C})-11(\mathrm{C})$ & 110.708 & $18(\mathrm{H})-17(\mathrm{C})$ & 1.093 \\
\hline $\begin{array}{c}15(\mathrm{H})-13(\mathrm{C})- \\
11(\mathrm{C})-10(\mathrm{O})\end{array}$ & -59.563 & $14(\mathrm{H})-13(\mathrm{C})-11(\mathrm{C})$ & 110.781 & $17(\mathrm{C})-11(\mathrm{C})$ & 1.530 \\
\hline $\begin{array}{c}13(\mathrm{C})-11(\mathrm{C})- \\
17(\mathrm{C})-20(\mathrm{H})\end{array}$ & 62.238 & $17(\mathrm{C})-11(\mathrm{C})-13(\mathrm{C})$ & 112.218 & $11(\mathrm{C})-13(\mathrm{C})$ & 1.523 \\
\hline $\begin{array}{c}13(\mathrm{C})-11(\mathrm{C})- \\
10(\mathrm{O})-2(\mathrm{C})\end{array}$ & -153.714 & $17(\mathrm{C})-11(\mathrm{C})-10(\mathrm{O})$ & 110.804 & $13(\mathrm{C})-14(\mathrm{H})$ & 1.093 \\
\hline $\begin{array}{c}11(\mathrm{C})-10(\mathrm{O})- \\
2(\mathrm{C})-21(\mathrm{H})\end{array}$ & -36.240 & $11(\mathrm{C})-10(\mathrm{O})-2(\mathrm{C})$ & 116.255 & $11(\mathrm{C})-12(\mathrm{H})$ & 1.099 \\
\hline $\begin{array}{c}11(\mathrm{C})-10(\mathrm{O})- \\
2(\mathrm{C})-1(\mathrm{C})\end{array}$ & -153.685 & $13(\mathrm{C})-11(\mathrm{C})-10(\mathrm{O})$ & 106.548 & $11(\mathrm{C})-10(\mathrm{O})$ & 1.433 \\
\hline $\begin{array}{c}11(\mathrm{C})-10(\mathrm{O})- \\
2(\mathrm{C})-6(\mathrm{C})\end{array}$ & 83.784 & $12(\mathrm{H})-11(\mathrm{C})-10(\mathrm{O})$ & 109.138 & $10(\mathrm{O})-2(\mathrm{C})$ & 1.433 \\
\hline $\begin{array}{c}10(\mathrm{O})-2(\mathrm{C})- \\
1(\mathrm{C})-5(\mathrm{H})\end{array}$ & -179.507 & $10(\mathrm{O})-2(\mathrm{C})-21(\mathrm{H})$ & 109.142 & $2(\mathrm{C})-21(\mathrm{H})$ & 1.099 \\
\hline $\begin{array}{c}10(\mathrm{O})-2(\mathrm{C})- \\
6(\mathrm{C})-9(\mathrm{H})\end{array}$ & 61.566 & $10(\mathrm{O})-2(\mathrm{C})-6(\mathrm{C})$ & 110.804 & $6(\mathrm{C})-9(\mathrm{H})$ & 1.093 \\
\hline $\begin{array}{c}10(\mathrm{O})-2(\mathrm{C})- \\
1(\mathrm{C})-4(\mathrm{H})\end{array}$ & 59.906 & $10(\mathrm{O})-2(\mathrm{C})-1(\mathrm{C})$ & 106.853 & $2(\mathrm{C})-1(\mathrm{C})$ & 1.523 \\
\hline $\begin{array}{c}11(\mathrm{C})-10(\mathrm{O})- \\
2(\mathrm{C})-1(\mathrm{C})\end{array}$ & -153.685 & $2(\mathrm{C})-1(\mathrm{C})-5(\mathrm{H})$ & 110.778 & $1(\mathrm{C})-4(\mathrm{H})$ & 1.092 \\
\hline
\end{tabular}

*Computed at DFT(B3LYP)/6311++G(d,p) level

\section{CONCLUSION}

In this study, the basic state optimized structures of some oxygenic fuel additives were calculated at the level of 6-311++ G (d,p) with the DFT (B3LYP) method. Methanol, ethanol, MTBE, ETBE, TAEE, TAME, and DIPE were used as fuel additives. The geometrical parameters of the fuel additives were determined and a detailed comparison of their physical and chemical properties was made. The effects of molecular energy and bond structure between $\mathrm{C}, \mathrm{H}$ and $\mathrm{O}$ on the chemical and physical properties of some oxygen fuel additives were investigated. It has been determined that there is a relationship between the molecular energy values of the fuel additives and the known octane numbers. The octane numbers of all the studied additives are close and the highest is approximately 115 for MTBE. The basic energy levels of all studied fuel additives were calculated and reported. MTBE, ETBE, TAEE, TAME and DIPE are often used as an additive for gasoline. In general, these additives have a common trend in reducing harmful emissions. It is not possible to give an exact value of emission reduction regarding the type and percentage of the additive in the fuel of $\mathrm{CO}, \mathrm{NO}_{\mathrm{x}}$ and $\mathrm{HC}$ emissions, because operating conditions, engine geometry and many other parameters are involved. However, an addition of these additives always leads to reduced $\mathrm{CO}$ emissions while there are no consistent results available about $\mathrm{NO}_{\mathrm{x}}$ emissions.

\section{REFERENCES}

[1] A. Demirbas, Green energy and technology: Biofuels, Springer, 2009

[2] EIA, Annual energy outlook 2012, with projections to 2035, U.S. Energy Information Administration, 2012

[3] BP, "BP Statistical review of world energy", available at: https://www.bp.com/en/global/corporate/energy-economics/statisticalreview-of-world-energy.html, 2019

[4] EPDK, Enerji yatirimcisi el kitabi, pp. 45-52, EPDK, 2012 (in Turkish) 
[5] PETDER, Petrol sektor raporu Ocak-Haziran, pp. 3-6, PETDER, 2012 (in Turkish)

[6] TPAO, Ham petrol ve dogal gaz sektor raporu”, TPAO, 2011 (in Turkish)

[7] EPDK, Petrol piyasası sekor raporu”, EPDK, 2012 (in Turkish)

[8] World Wildlife Fund, Enerji raporu, WWF, 2011 (in Turkish)

[9] International Energy Agency, Key world energy statistics, IEA, 2012

[10] Organization of the Petroleum Exporting Countries, World oil outlook, OPEC, 2012

[11] A. Arteconi, A. Mazzarini, G. D. Nicola, "Emissions from ethers and organic carbonate fuel additives: A review", Water, Air, \& Soil Pollution, Vol. 221, Article ID 405, 2011

[12] O. I. Awad, R. Mamat, O. M. Ali, N. A. C. Sidik, T. Yusaf, K. Kadirgama, M. Kettner, "Alcohol and ether as alternative fuels in spark ignition engine: A review", Renewable and Sustainable Energy Reviews, Vol. 82, pp. 2586-2605, 2018

[13] H. Sharudin, N. R. Abdullah, G. Najafi, R. Mamat, H. H. Masjuki, "Investigation of the effects of iso-butanol additives on spark ignition engine fuelled with methanol-gasoline blends", Applied Thermal Engineering, Vol. 114, pp. 593-600, 2017

[14] I. Sezer, A. Bilgin, "Effects of methyl tert-butyl ether addition to base gasoline on the performance and $\mathrm{CO}$ emissions of a spark ignition engine”, Energy Fuels, Vol. 22, No. 2, pp. 1341-1348, 2008

[15] M. K. Balki, C. Sayin, M. Canakci, "The effect of different alcohol fuels on the performance, emission and combustion characteristics of a gasoline engine", Fuel, Vol. 115, pp. 901-906, 2014

[16] W. D. Hsieh, R. H. Chen, T. L. Wu, T. H. Lin, "Engine performance and pollutant emission of an SI engine using ethanol-gasoline blended fuels", Atmospheric Environment, Vol. 36, No. 3, pp. 403-410, 2002

[17] M. Canakci, A. N. Ozsezen, E. Alptekin, M. Eyidogan, "Impact of alcohol-gasoline fuel blends on the exhaust emission of an SI engine", Renewable Energy, Vol. 52, pp. 111-117, 2013

[18] Z. Muzikova, M. Pospisil, G. Sebor, "Volatility and phase stability of petrol blends with ethanol", Fuel, Vol. 88, No. 8, pp. 1351-1356, 2009

[19] V. R. Surisetty, A. K. Dalai, J. Kozinski, “Alcohols as alternative fuels: An overview”, Applied Catalysis A: General, Vol. 404, No. 1-2, pp. 111,2011

[20] E. W. D. Menezes, R. Cataluna, D. Samios, R. D. Silva, "Addition of an azeotropic ETBE/ethanol mixture in eurosuper-type gasolines", Fuel, Vol. 85, No. 17-18, pp. 2567-2577, 2006

[21] D. P. Lince, L. R. Wilson, G. A. Carlson, "Methyl tert-butyl ether (MTBE) contamination in private wells near gasoline stations in upstate New York", Bulletin of Environmental Contamination and Toxicology, Vol. 61, No. 4, pp. 484-488, 1998

[22] F. Ancillotti, V. Fattore, "Oxygenate fuels: Market expansion and catalytic aspect of synthesis", Fuel Processing Technology, Vol. 57, No. 3, pp. 163-194, 1998

[23] E. W. D. Menezes, R. Cataluna, "Optimization of the ETBE (ethyl tertbutyl ether) production process", Fuel Processing Technology, Vol. 89, No. 11, pp. 1148-1152, 2008

[24] R. D. Silva, R. Cataluna, E. W. D. Menezes, D. Samios, C. M. S Piatnicki, "Effect of additives on the antiknock properties and Reid vapor pressure of gasoline", Fuel, Vol. 84, No. 7-8, pp. 951-959, 2005

[25] C. Vauclair, H. Tarjus, P. Schaetzel, "Permselective properties of PVAPAA blended membrane used for dehydration of fusel oil by pervaporation”, Journal of Membrane Science, Vol. 125, No. 2, pp. 293301, 1997

[26] R. Cataluna, R. D. Silva, E. W. D. Menezes, R. B. Ivanov, "Specific consumption of liquid biofuels in gasoline fuelled engines", Fuel, Vol. 87, No. 15-16, pp. 3362-3368, 2008

[27] S. Miertus, E. Scrocco, J. Tomasi, "Electrostatic interaction of a solute with a continuum. A direct utilizaion of $\mathrm{AB}$ initio molecular potentials for the prevision of solvent effects", Chemical Physics, Vol. 55, No. 1, pp. 117-129, 1981

[28] R. Cammi, J. Tomasi, "Remarks on the use of the apparent surface charges (ASC) methods in solvation problems: Iterative versus matrix- inversion procedures and the renormalization of the apparent charges", Journal of Computational Chemistry, Vol. 16, No. 12, pp. 1449-1458, 1995

[29] M. J. Frisch, G. Trucks, K. Schlegel, G. Scuseria, M. Robb, J Cheeseman, J. Montgomery, T. Vreven, K. N. Kudin, J. Burant, J Millam, S. Iyengar, J. Tomasi, V. Barone, B. Mennucci, M. Cossi, G. Scalmani, N. Rega, A. Petersson, H. Nakatsuji, M. Hada, M. Ehara, K. Toyota, R. Fukuda, J. Hasegawa, M. Ishida, T. Nakajima, Y. Honda, O. Kitao, H. Nakai, M. Klene, X. Li, J. Knox, H. Hratchian, D. Cross, V. Bakken, C. Adamo, J. J. Merchan, R. Gomperts, R. Stratmann, O. Yazyev, A. Austin, R. Cammi, C. Pomelli, J. Ochterski, P. Ayala, K. Morokuma, G. Voth, P. Salvador, J. J. Dannenberg, V. G. Zakrzewski, S. Dapprich, A. D. Daniels, M. Strain, O. Farkas, S. Malick, A. Rabuck, K. Raghavachari, J. Foresman, J. Ortiz, Q. Cui, A. G. Baboul, S. Clifford, J. Cioslowski, B. Stefanov, G. Liu, A. Liashenko, P. Piskorz, I. Komaromi, R. Mata, D. Fox, T. Keith, S. Laham, C. Y. Peng, A. Nanayakkara, M. Challacombe, P. Gill, B. Johnson, W. Chen, M. Wong, R. S. Gonzalez, J. Pople, J. Dannenberg, V. Zakrzewski, A. Daniels, A. G. Baboul, Y. Peng, G. E. Scuseria, J. M. Millam, J. B. Foresman, M. J. Frisch, J. A. Montgomery, R. E. Stratmann, D. K. Malick, X. B. Li, T. Keith, M. W. Wong, H. B. Schlegel, P. Y. Ayala, B. B. Stefanov, M. Hada, R. L. Martin, K. N. Kudin, H. P. Hratchian, G. A. Voth, A. J. Austin, M. C. Strain, C. Adamo, P. M. W. Gill, M. A. Robb, G. A. Petersson, J. B. Cross, J. L. Torre, G. W. Trucks, J. C. Burant, D. J. Fox, A. D. Rabuck, C. Huerta, M. Akhras, J. R. Cheeseman, S. S. Iyengar, J. A. Pople, J. E. Knox, J. W. Ochterski, B. A. Johnson, Gaussian 03. Revision C.02, Gaussian Inc., 2004

[30] A. Frish, A. B. Nielsen, A. J. Holder, Gauss view user manual, Gaussian Inc., 2001 